\title{
Application of Jacobi Algorithm for ISI Channels*
}

\author{
Pei Xiao and Mathini Sellathurai \\ The Institute of Electronics, Communications and Information Technology, \\ Queen's University Belfast, BT3 9DT, United Kingdom. \\ E-mail: \{pei.xiao, m.sellathurai\}@ecit.qub.ac.uk
}

\begin{abstract}
In this paper, we apply the Jacobi iterative algorithm to combat intersymbol interference (ISI) caused by frequency selective channels. The performance bound of the equalizer is analyzed in order to gain an insight into its asymptotic behavior. Due to the error propagation problem, the potential of this algorithm is not reached in an uncoded system. However, its extension to a coded system with the application of the turbo processing principle results in a new turbo equalization algorithm which demonstrates comparable performance with reduced complexity compared to some existing filter based turbo equalization schemes; and superior performance compared to some frequency domain solutions, such as orthogonal frequency division multiplexing (OFDM) and single-carrier frequency domain equalization (SC-FDE).
\end{abstract}

\section{INTRODUCTION}

In a cellular mobile communications environment, multipath propagation causes dispersion of transmitted signals. The time delay spread causes intersymbol interference (ISI) and degrades system performance. Orthogonal frequency division multiplexing (OFDM) technique provides an effective means to combat multipath fading since it divides the transmission bandwidth into many narrow bands, each of which exhibits an approximately flat fading. Another effective remedy to mitigate the effects of ISI is the use of equalization methods. A frequency selective channel can be described as a rate one convolutional code defined over the field of real or complex numbers, so the combination of a channel code and an ISI channel can be viewed as a serially concatenated system, and can be decoded using a turbo processing principle. Douillard et al. proposed turbo equalization scheme in [1] which combines equalization with channel decoding to remove the effect of ISI. It is shown that turbo equalization significantly improves the performance over separate equalization and decoding. In its original form, turbo equalization employed the maximum a posteriori probability (MAP) algorithm for both equalization and decoding. For channels with

\footnotetext{
${ }^{*}$ This work has been presented in part at the IEEE International Conference on Communications (ICC) 2008.
} 
large delay spreads and for large constellation sizes, it suffers from prohibitive computational complexity due to the increasing number of trellis states. In [2]-[6], etc., the MAP-equalizer was replaced by a linear filter, whose coefficients are adjusted to minimize the mean-square error. It was shown that the performance of this approach is similar to that of the MAP-based receiver, while providing a significant reduction in the computational complexity, especially when the MMSE linear filter is derived under the constraint of the perfect a priori information resulting in an approximate implementation of the original MMSE based scheme [3], [6].

In this paper, we first design an equalizer based on the Jacobi iterative algorithm. The algorithm is then extended to a coded system, leading to a new approach to turbo equalization, which further reduces receiver complexity without incurring a performance penalty compared to most existing filter based algorithms. The remainder of this paper is structured as follows. The application of the Jacobi algorithm in ISI channels is discussed in Section II, and is extended to turbo equalization in a coded system in Section III. The simulation and analytical results of the proposed turbo equalization algorithm are shown; the performance and complexity of the proposed turbo equalization scheme are compared with some existing filter based schemes as well as OFDM [7], [8] solution and single-carrier frequency domain equalization (SC-FDE) [8], [9] in Section IV. Conclusions are drawn in Section V. The performance bound for the proposed equalization, turbo equalization are theoretically analyzed in Section II-C, III-B, respectively.

\section{Equalization BAsed on Jacobi Algorithm}

\section{A. Problem formulation}

A multipath channel can be modeled by an equivalent baseband system where the transmit filter, the channel and the receive filter, are represented by a discrete-time $L$-tap transversal filter with finite-length

impulse response $h_{n}=\sum_{l=0}^{L-1} h_{l} \delta_{n-l}$ where $h_{l}$ denotes the complex channel coefficients, which are normalized such that $\sum_{l=0}^{L-1}\left|h_{l}\right|^{2}=1$. The received signal can be formed as

$$
r_{n}=\sum_{l=0}^{L-1} s_{n-l} h_{l}+v_{n},
$$

where $s_{n}=x_{n}+j y_{n}$ denotes the transmitted PSK/QAM symbol at time instant $n$, and $v_{n}$ is the complex additive white Gaussian noise (AWGN) with zero mean and variance $N_{0}$. We choose the QPSK modulation scheme to simplify the algorithm development. However, the proposed algorithm can be readily extended to higher constellation PSK or QAM systems. The task of the receiver is to detect the transmitted symbols $\left\{s_{n}\right\}$ given the received observation $\left\{r_{n}\right\}$. From (1), we see that the desired symbol is corrupted with ISI and AWGN. An equalizer is needed to combat ISI. Several equalization algorithms have been introduced in the literature, e.g., the minimum mean square error (MMSE) linear equalizer, and the decision feedback equalizer (DFE). Various adaptive algorithms have been proposed for equalizer training, e.g., the least mean square (LMS), and the recursive least square (RLS) [10], square root Kalman 
(SRK) [11], etc.. Here, we introduce a new equalization algorithm and derive its theoretical performance bound, and our analysis reveals its good potential for removing the detrimental effect of ISI.

To simplify the algorithm derivation, we use a 3-tap broadband fixed wireless access (BFWA) channel specified in IEEE 802.16 standard [12] as an example. The algorithm can be easily extended to address generic ISI channels. Tailored for different terrain conditions, a set of 6 typical channel models called Stanford University Interim (SUI) Channel Models were proposed in [13] for simulation, design, development and testing of technologies suitable for BFWA applications. All of them are simulated using 3 taps, having either Ricean or Rayleigh amplitude distributions. For the purpose of this study, we select the SUI3 channel which has a tap spacing of 500ns, and maximum tap delay of 1000ns. Under the assumption that the transmitted data rate is $4 \mathrm{Mbps}$, the multipath fading can be modeled as a tapped-delay line with adjacent taps spaced equally at the symbol rate. The received signal is formed as

$$
r_{n}=h_{0} s_{n}+h_{1} s_{n-1}+h_{2} s_{n-2}+v_{n}
$$

where the channel coefficients $h_{0}, h_{1}, h_{2}$ are complex Gaussian random variables and are assumed to remain constant during the transmission of one block of data.

\section{B. Algorithm derivation}

Based on (2), the received signal can be written in vector form as

$$
\underbrace{\left[\begin{array}{c}
r_{n} \\
r_{n+1} \\
r_{n+2}
\end{array}\right]}_{\mathbf{r}_{n}}=\underbrace{\left[\begin{array}{ccccc}
h_{2} & h_{1} & h_{0} & 0 & 0 \\
0 & h_{2} & h_{1} & h_{0} & 0 \\
0 & 0 & h_{2} & h_{1} & h_{0}
\end{array}\right]}_{\mathbf{H}} \underbrace{\left[\begin{array}{c}
s_{n-2} \\
s_{n-1} \\
s_{n} \\
s_{n+1} \\
s_{n+2}
\end{array}\right]}_{\mathbf{s}_{n}}+\underbrace{\left[\begin{array}{c}
v_{n} \\
v_{n+1} \\
v_{n+2}
\end{array}\right]}_{\mathbf{v}_{n}} .
$$

The least square estimate of the symbol vector $\mathbf{s}_{n}$ is [14]

$$
\hat{\mathbf{s}}_{n}=\left(\mathbf{H}^{*} \mathbf{H}\right)^{-1} \mathbf{H}^{*} \mathbf{r}_{n}=\left(\mathbf{H}^{*} \mathbf{H}\right)^{-1} \mathbf{H}^{*}\left(\mathbf{H} \mathbf{s}_{n}+\mathbf{v}_{n}\right)=\mathbf{s}_{n}+\left(\mathbf{H}^{*} \mathbf{H}\right)^{-1} \mathbf{H}^{*} \mathbf{v}_{n},
$$

which is an unbiased estimate of $\mathbf{s}_{n}$ since $\mathrm{E}\left[\hat{\mathbf{s}}_{n}\right]=\mathbf{s}_{n}$. However, this procedure is computationally complex due to the matrix inverse operation for each symbol vector. The superscript operator ()$^{*}$ is the conjugate transpose operation when applied to matrices, and simply the conjugate when applied to scalars. To simplify the computation, let us reform (4) as

$$
\hat{\mathbf{s}}_{n}=\mathbf{s}_{n}+\left(\mathbf{H}^{*} \mathbf{H}\right)^{-1} \mathbf{H}^{*} \mathbf{v}_{n}=\mathbf{s}_{n}+\mathbf{R}^{-1} \mathbf{u}_{n}=\mathbf{R}^{-1}\left(\mathbf{R} \mathbf{s}_{n}+\mathbf{u}_{n}\right)=\mathbf{R}^{-1} \mathbf{y}_{n},
$$


where

$$
\begin{aligned}
& \mathbf{R}=\mathbf{H}^{*} \mathbf{H}=\left[\begin{array}{ccccc}
\left|h_{2}\right|^{2} & h_{2}^{*} h_{1} & h_{2}^{*} h_{0} & 0 & 0 \\
h_{1}^{*} h_{2} & \left|h_{1}\right|^{2}+\left|h_{2}\right|^{2} & h_{1}^{*} h_{0}+h_{2}^{*} h_{1} & h_{2}^{*} h_{0} & 0 \\
h_{0}^{*} h_{2} & h_{0}^{*} h_{1}+h_{1}^{*} h_{2} & \left|h_{0}\right|^{2}+\left|h_{1}\right|^{2}+\left|h_{2}\right|^{2} & h_{1}^{*} h_{0}+h_{2}^{*} h_{1} & h_{2}^{*} h_{0} \\
0 & h_{0}^{*} h_{2} & h_{0}^{*} h_{1}+h_{1}^{*} h_{2} & \left|h_{0}\right|^{2}+\left|h_{1}\right|^{2} & h_{1}^{*} h_{0} \\
0 & 0 & h_{0}^{*} h_{2} & h_{0}^{*} h_{1} & \left|h_{0}\right|^{2}
\end{array}\right] ; \\
& \mathbf{u}_{n}=\mathbf{H}^{*} \mathbf{v}_{n} ; \\
& \mathbf{y}_{n}=\mathbf{R s}_{n}+\mathbf{u}_{n}=\mathbf{H}^{*} \mathbf{H s}_{n}+\mathbf{H}^{*} \mathbf{v}_{n}=\mathbf{H}^{*} \mathbf{r}_{n} .
\end{aligned}
$$

Note that the matrix inverse $\mathbf{R}^{-1}$ can be pre-computed for static ISI channels. However, direct implementation of (5), i.e., zero forcing solution leads to noise enhancement problem. Apparently, $\mathbf{R}$ is a Hermitian matrix satisfying the condition $\mathbf{R}=\mathbf{R}^{*}$. Let us decompose the matrix $\mathbf{R}$ into 2 matrices $\mathbf{R}=\mathbf{D}+\mathbf{R}_{\text {off }}$, where $\mathbf{D}$ is a diagonal matrix, and $\mathbf{R}_{\text {off }}$ is an off-diagonal matrix. For the matrix $\mathbf{R}$ expressed in (6), $\mathbf{D}$ and $\mathbf{R}_{\text {off }}$ are

$$
\begin{aligned}
\mathbf{D} & =\operatorname{diag}\left\{\left|h_{2}\right|^{2},\left|h_{1}\right|^{2}+\left|h_{2}\right|^{2},\left|h_{0}\right|^{2}+\left|h_{1}\right|^{2}+\left|h_{2}\right|^{2},\left|h_{0}\right|^{2}+\left|h_{1}\right|^{2},\left|h_{0}\right|^{2}\right\} \\
\mathbf{R}_{\text {off }} & =\left[\begin{array}{ccccc}
0 & h_{2}^{*} h_{1} & h_{2}^{*} h_{0} & 0 & 0 \\
h_{1}^{*} h_{2} & 0 & h_{1}^{*} h_{0}+h_{2}^{*} h_{1} & h_{2}^{*} h_{0} & 0 \\
h_{0}^{*} h_{2} & h_{0}^{*} h_{1}+h_{1}^{*} h_{2} & 0 & h_{1}^{*} h_{0}+h_{2}^{*} h_{1} & h_{2}^{*} h_{0} \\
0 & h_{0}^{*} h_{2} & h_{0}^{*} h_{1}+h_{1}^{*} h_{2} & 0 & h_{1}^{*} h_{0} \\
0 & 0 & h_{0}^{*} h_{2} & h_{0}^{*} h_{1} & 0
\end{array}\right] .
\end{aligned}
$$

The matrix inversion in (5) can be solved iteratively by the Jacobi algorithm [15]

$$
\mathbf{s}_{n}^{(i)}=\mathbf{D}^{-1}\left(\mathbf{y}_{n}-\mathbf{R}_{\mathrm{off}} \mathbf{s}_{n}^{(i-1)}\right)
$$

where $i$ is the iteration index. Substituting (7) into (8) yields

$$
\begin{gathered}
\mathbf{D}^{-1}=\operatorname{diag}\left\{1 /\left|h_{2}\right|^{2}, 1 /\left(\left|h_{1}\right|^{2}+\left|h_{2}\right|^{2}\right), 1 /\left(\left|h_{0}\right|^{2}+\left|h_{1}\right|^{2}+\left|h_{2}\right|^{2}\right), 1 /\left(\left|h_{0}\right|^{2}+\left|h_{1}\right|^{2}\right), 1 /\left|h_{0}\right|^{2}\right\} \\
{\left[\begin{array}{c}
s_{n-2}^{(i)} \\
s_{n-1}^{(i)} \\
s_{n}^{(i)} \\
s_{n+1}^{(i)} \\
s_{n+2}^{(i)}
\end{array}\right]=\mathbf{D}^{-1}\left\{\left[\begin{array}{c}
h_{2}^{*} r_{n} \\
h_{2}^{*} r_{n}+h_{2}^{*} r_{n+1} s_{n-1}^{(i-1)}+h_{2}^{*} h_{0} s_{n}^{(i-1)} \\
h_{0}^{*} r_{n}+h_{1}^{*} r_{n+1}+h_{2}^{*} r_{n+2} \\
h_{0}^{*} r_{n+1}+h_{1}^{*} r_{n+2} \\
h_{0}^{*} r_{n+2}
\end{array}\right]-\left[\begin{array}{c}
(i-1) \\
h_{1}^{*} h_{2} s_{n-2}^{(i-1)}+\left(h_{1}^{*} h_{0}+h_{2}^{*} h_{1}\right) s_{n}^{(i-1)}+h_{2}^{*} h_{0} s_{n+1}^{(i-1)} \\
h_{0}^{*} h_{2} s_{n-2}^{(i-1)}+\left(h_{0}^{*} h_{1}+h_{1}^{*} h_{2}\right) s_{n-1}^{(i-1)}+\left(h_{1}^{*} h_{0}+h_{2}^{*} h_{1}\right) s_{n+1}^{(i-1)}+h_{2}^{*} h_{0} s_{n+2}^{(i-1)} \\
h_{0}^{*} h_{2} s_{n-1}^{(i-1)}+\left(h_{0}^{*} h_{1}+h_{1}^{*} h_{2}\right) s_{n}^{(i-1)}+h_{1}^{*} h_{0} s_{n+2}^{(i-1)} \\
h_{0}^{*} h_{2} s_{n}^{(i-1)}+h_{0}^{*} h_{1} s_{n+1}^{(i-1)}
\end{array}\right\}\right.}
\end{gathered}
$$

The above procedure produces estimates of all the symbols in the vector $\mathbf{s}_{n}^{(i)}$. However, since detection of the central symbol $s_{n}$ relies on all the received samples in the vector $\mathbf{y}_{n}\left(\mathbf{r}_{n}\right)$, it can be assumed more 
accurate than the detection of the other symbols. From the above equation, we derive

$$
\begin{aligned}
z_{n}= & s_{n}^{(i)}=\left[h_{0}^{*} r_{n}+h_{1}^{*} r_{n+1}+h_{2}^{*} r_{n+2}-h_{0}^{*} h_{2} s_{n-2}^{(i-1)}-\left(h_{0}^{*} h_{1}+h_{1}^{*} h_{2}\right) s_{n-1}^{(i-1)}-\left(h_{1}^{*} h_{0}+h_{2}^{*} h_{1}\right) s_{n+1}^{(i-1)}-h_{2}^{*} h_{0} s_{n+2}^{(i-1)}\right] / \mathcal{P} \\
= & {\left[h_{0}^{*}\left(r_{n}-h_{2} s_{n-2}^{(i-1)}-h_{1} s_{n-1}^{(i-1)}\right)+h_{1}^{*}\left(r_{n+1}-h_{2} s_{n-1}^{(i-1)}-h_{0} s_{n+1}^{(i-1)}\right)+h_{2}^{*}\left(r_{n+2}-h_{1} s_{n+1}^{(i-1)}-h_{0} s_{n+2}^{(i-1)}\right)\right] / \mathcal{P} } \\
= & s_{n}+\underbrace{\left[h_{0}^{*} h_{2}\left(s_{n-2}-s_{n-2}^{(i-1)}\right)+\left(h_{0}^{*} h_{1}+h_{1}^{*} h_{2}\right)\left(s_{n-1}-s_{n-1}^{(i-1)}\right)+\left(h_{1}^{*} h_{0}+h_{2}^{*} h_{1}\right)\left(s_{n+1}-s_{n+1}^{(i-1)}\right)+h_{2}^{*} h_{0}\left(s_{n+2}-s_{n+2}^{(i-1)}\right)\right] / \mathcal{P}}_{\text {cancellation residual }} \\
& +\underbrace{\left[h_{0}^{*} v_{n}+h_{1}^{*} v_{n+1}+h_{2}^{*} v_{n+2}\right] / \mathcal{P}}_{\text {noise }},
\end{aligned}
$$

where $\mathcal{P}=\sum_{i=0}^{2}\left|h_{i}\right|^{2}$. One can see from (9) that the decision statistic $z_{n}$ for the symbol $s_{n}$ at the $i^{t h}$ iteration is obtained by canceling the interference using the symbol estimates at the $(i-1)^{t h}$ iteration. Note that at the beginning of the iterative process, no symbol estimates are available. We can use either coherent detector or conventional linear MMSE equalizer to obtain an initial estimate of the transmitted symbols so that the interference cancellation can be carried out in the subsequent stages ${ }^{1}$. To detect the transmitted symbols coherently, we correct the phase shift by multiplying the received signal with the conjugate of $\hat{h}_{0}$ before making a symbol decision, i.e.,

$$
z_{n}=\hat{h}_{0}^{*} r_{n}=\hat{h}_{0}^{*}\left(h_{0} s_{n}+h_{1} s_{n-1}+h_{2} s_{n-2}+v_{n}\right)=\hat{h}_{0}^{*} h_{0} s_{n}+\underbrace{\hat{h}_{0}^{*}\left(h_{1} s_{n-1}+h_{2} s_{n-2}+v_{n}\right)}_{\text {combined ISI and noise }},
$$

where $\hat{h}_{0}$ is an estimate of $h_{0}$. Alternatively, we can use a linear MMSE equalizer [10] for initial symbol estimates. Assuming the equalizer has $2 t+1$ taps and the detection delay $d$, it is designed to minimize the mean square error (MSE) between the equalizer output $z_{n}$ and the symbol $s_{n-d}$, i.e., $e=\mathrm{E}\left\{\left|z_{n}-s_{n-d}\right|^{2}\right\}=\mathrm{E}\left\{\left|\mathbf{c}^{*} \mathbf{r}_{n}-s_{n-d}\right|^{2}\right.$, where $\mathrm{E}(\cdot)$ denotes expectation (statistical averaging). The output $z_{n}$ is formed as

$$
z_{n}=\sum_{k=0}^{2 t} c_{k}^{*} r_{n-k}=\mathbf{c}^{*} \mathbf{r}_{n}
$$

where $\mathbf{r}_{n}=\left[\begin{array}{llllll}r_{n} & r_{n-1} & \cdots & r_{n-2 t+1} & r_{n-2 t}\end{array}\right]^{T}$, and $\mathbf{c}=\left[\begin{array}{lllll}c_{0} & c_{1} & \cdots & c_{2 t-1} & c_{2 t}\end{array}\right]^{T}$, where the superscript operator ()$^{T}$ stands for transpose operation. The coefficients vector is computed as

$$
\mathbf{c}=\left(\mathrm{E}\left[\mathbf{r}_{n} \mathbf{r}_{n}^{*}\right]\right)^{-1}\left(\mathrm{E}\left[\mathbf{r}_{n}^{*} s_{n-d}\right]\right)^{*}=\mathbf{R}^{-1} \mathbf{p}^{*}
$$

where $\mathbf{p}=\mathrm{E}\left[\mathbf{r}_{n}^{*} s_{n-d}\right]$ is the cross-correlation vector, and $\mathbf{R}^{-1}$ is the inverse of the autocorrelation matrix $\mathbf{R}=\mathrm{E}\left[\mathbf{r}_{n} \mathbf{r}_{n}^{*}\right]$. Once the decision statistic $z_{n}$ is obtained by coherent detection or MMSE equalization, the initial symbol estimate can be derived using maximum likelihood decision rule, i.e.,

$$
s_{n}^{(0)}=\arg \min _{s_{m} \in\left\{s_{0}, s_{1}, s_{2}, s_{3}\right\}}\left|z_{n}-s_{m}\right|^{2}=\arg \max _{s_{m}} \operatorname{Re}\left\{s_{m}^{*} z_{n}\right\} .
$$

\footnotetext{
${ }^{1}$ The word "stage" is used interchangeably with "iteration" in this paper.
} 


\section{Performance bound of the proposed equalizer}

To find out the theoretical potential of the proposed approach, we analyze the performance bound that can be achieved by the equalization scheme expressed by (9). It is derived based on the assumption of perfect channel estimation and perfect cancellation. In this case, all the cancellation residuals will vanish, the equalizer output $z_{n}$ in (9) only contains the desired signal and the noise, i.e.,

$$
z_{n}=s_{n}+\frac{1}{\mathcal{P}} \sum_{l=0}^{L-1} h_{l}^{*} v_{n-l}=s_{n}+w_{n},
$$

where $L$ is the number of channel taps, $\mathcal{P}=\sum_{l=0}^{L-1}\left|h_{l}\right|^{2}$, and $w_{n}=\frac{1}{\mathcal{P}} \sum_{l=0}^{L-1} h_{l}^{*} v_{n-l} \sim \mathcal{C N}\left(0, N_{0} / \mathcal{P}\right)$. For the QPSK signals, the bit error probability is computed as [16]

$$
P_{b}=Q\left(\frac{\sqrt{E_{b} \sum_{l=0}^{L-1}\left|h_{l}\right|^{2}}}{\sqrt{N_{0} / 2}}\right)=Q\left(\sqrt{\frac{2 E_{b} \mathcal{P}}{N_{0}}}\right),
$$

where $Q(x)=\int_{x}^{\infty} \frac{1}{\sqrt{2 \pi}} \exp \left(-t^{2} / 2\right) d t$ is the complementary Gaussian cumulative distribution function.

The above formula can be used directly for calculating the performance bound for static channels. Next, we use the SUI-3 channel as an example to demonstrate how the performance bound for nonstatic channels can be calculated. For the 3-tap SUI-3 channel, $\mathcal{P}=\left|h_{0}\right|^{2}+\left|h_{1}\right|^{2}+\left|h_{2}\right|^{2}$. Let us denote $x=\left|h_{0}\right|^{2}, y=\left|h_{1}\right|^{2}+\left|h_{2}\right|^{2}$. The bit error probability is a function of random variables $x$ and $y$, i.e.,

$$
P_{b}(x, y)=Q\left(\sqrt{\frac{2 E_{b}(x+y)}{N_{0}}}\right) .
$$

Since $\left|h_{0}\right|$, the amplitude of the first tap is Ricean distributed due to the existence of line of sight propagation, the random variable $x$ is non-central chi-square distributed with 2 degrees of freedom and PDF

$$
p(x)=\frac{1}{2 \sigma^{2}} \exp \left(-\frac{x+s^{2}}{2 \sigma^{2}}\right) I_{0}\left(\frac{\sqrt{x} s}{\sigma^{2}}\right), \quad x \geq 0,
$$

where $I_{0}(x)$ is the $0^{t h}$ order modified Bessel function of the first kind [10, p. 44]. The amplitudes of the other two taps $\left(\left|h_{1}\right|,\left|h_{2}\right|\right)$ are characterized by a Rayleigh distribution. Therefore, each of the random variables $\left|h_{1}\right|^{2},\left|h_{2}\right|^{2}$ has a central chi-square distribution with 2 degrees of freedom and characteristic functions

$$
\begin{aligned}
& \psi_{\left|h_{1}\right|^{2}}(j v)=\left(1-j v \gamma_{1}\right)^{-1} \\
& \psi_{\left|h_{2}\right|^{2}}(j v)=\left(1-j v \gamma_{2}\right)^{-1},
\end{aligned}
$$

where $\gamma_{1}=\mathrm{E}\left[\left|h_{1}\right|^{2}\right], \gamma_{2}=\mathrm{E}\left[\left|h_{2}\right|^{2}\right]$, and $\gamma_{1} \neq \gamma_{2}$. As a consequence of the statistical independence of $\left|h_{1}\right|^{2}$ and $\left|h_{2}\right|^{2}$, the characteristic function of $y$ is

$$
\begin{aligned}
\psi_{y}(j v) & =\left(1-j v \gamma_{1}\right)^{-1}\left(1-j v \gamma_{2}\right)^{-1} \\
& =\left(1-\frac{\gamma_{2}}{\gamma_{1}}\right)^{-1}\left(1-j v \gamma_{1}\right)^{-1}+\left(1-\frac{\gamma_{1}}{\gamma_{2}}\right)^{-1}\left(1-j v \gamma_{2}\right)^{-1} \\
& =\frac{\gamma_{1}}{\gamma_{1}-\gamma_{2}}\left(1-j v \gamma_{1}\right)^{-1}+\frac{\gamma_{2}}{\gamma_{2}-\gamma_{1}}\left(1-j v \gamma_{2}\right)^{-1} .
\end{aligned}
$$


Taking the inverse Fourier transform, we obtain the PDF of $y$ as

$$
p(y)=\frac{1}{\gamma_{1}-\gamma_{2}} \exp \left(-\frac{y}{\gamma_{1}}\right)+\frac{1}{\gamma_{2}-\gamma_{1}} \exp \left(-\frac{y}{\gamma_{2}}\right), \quad y \geq 0 .
$$

To obtain the error probability when $x, y$ are random, we must average $P_{b}(x, y)$ expressed in (16) over the distribution of $x, y$, i.e., the average BER is calculated as

$$
\begin{aligned}
\bar{P}_{b}= & \int_{0}^{\infty} \int_{0}^{\infty} P_{b}(x, y) p(x) p(y) d y d x \\
= & \frac{1}{2 \sigma^{2}} \int_{0}^{\infty} \int_{0}^{\infty} Q\left(\sqrt{\frac{2 E_{b}(x+y)}{N_{0}}}\right) \exp \left(-\frac{x+s^{2}}{2 \sigma^{2}}\right) I_{0}\left(\frac{\sqrt{x} s}{\sigma^{2}}\right) \\
& \cdot\left[\frac{1}{\gamma_{1}-\gamma_{2}} \exp \left(-\frac{y}{\gamma_{1}}\right)+\frac{1}{\gamma_{2}-\gamma_{1}} \exp \left(-\frac{y}{\gamma_{2}}\right)\right] d y d x
\end{aligned}
$$

\section{Numerical results for the equalizer in an uncoded system}

Computer simulations are carried out to demonstrate the performance of the proposed algorithm. During each Monte-Carlo run, the block size is set to 10000 bits, which correspond to 5000 QPSK symbols, 200 of which are used as pilot symbols for channel estimation. This is conducted using the modified maximum likelihood algorithm presented in [17]. Both time-varying and static channels are tested. In the former case, we choose the SUI-3 BFWA channel. The channel coefficients vary from one data block to another, however, they are assumed to remain constant during the transmission of one block of data. The simulated results are averaged over 1000 channel realizations. For the static channels, we use the Proakis B channel [5]) with impulse response $h[n]=0.407 \delta[n]+0.815 \delta[n-1]+0.407 \delta[n-2]$, and the 5-tap channel [5] with impulse response $h[n]=(2-0.4 j) \delta[n]+(1.5+1.8 j) \delta[n-1]+\delta[n-2]+(1.2-$ $1.3 j) \delta[n-3]+(0.8+1.6 j) \delta[n-4]$. The channel is normalized so that $P=\sum_{n=0}^{4}|h[n]|^{2}=1$.

In Fig. 1 and Fig. 2, we show the performance of the two variants of the proposed algorithm (one is initialized with coherent detection, the other one is initialized with a 8-tap linear MMSE equalization as described in Section II-B). As indicated by both figures, the second scheme with the MMSE initialization yields much better performance. In Fig. 1, we also compare the performance of the proposed scheme with the conventional DFE equalizer with 5 feedforward, 3 feedback taps for the SUI-3 channel. It employs 200 pilots for training the equalizer coefficients, using recursive least square adaptation. Fig. 1 shows that the proposed equalizer initialized with an MMSE filter performs better than the DFE; whereas the DFE performs better than the proposed equalizer initialized with coherent detection at high SNR. We also see that the proposed scheme has a much lower performance bound than that of the DFE, thus exhibits a much better potential. This potential can be realized by extending the algorithm to turbo equalization as will be demonstrated in Section III and IV.

The performance of the proposed equalizer at different stages is depicted in Fig. 2 for the two static channels. Obviously, the improvement by applying the Jacobi iterative algorithm over coherent detection (MMSE equalization) is significant if we compare the topmost curve with the other curves. It takes only 


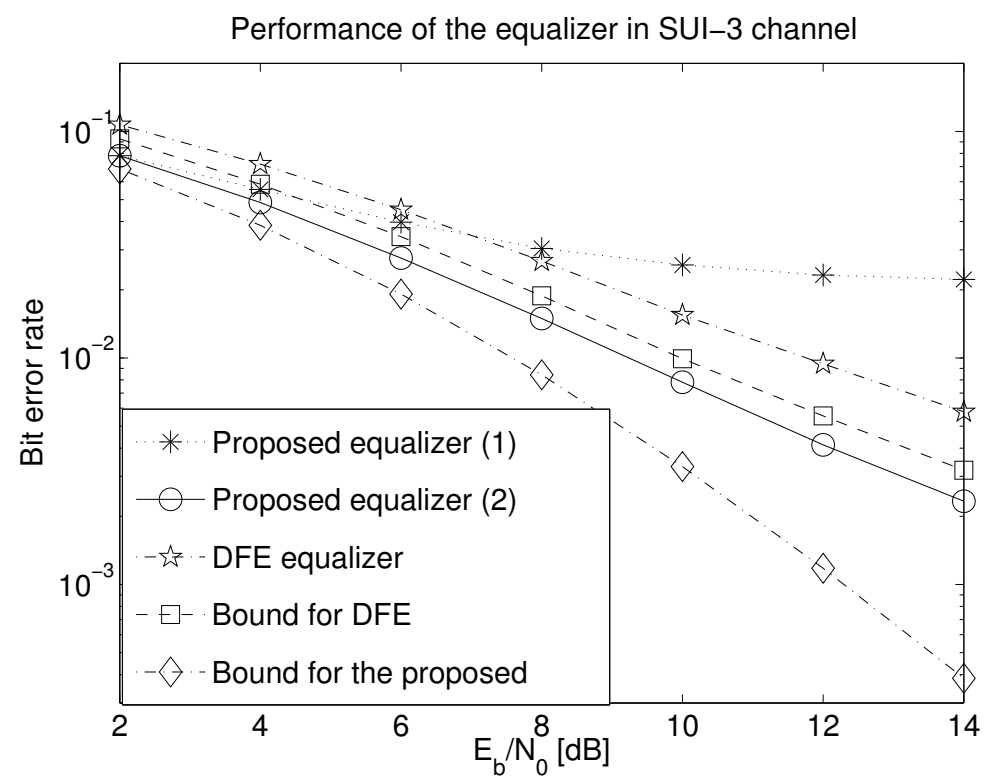

Fig. 1. Performance of the Jacobi algorithm for SUI-3 channel. The curves corresponding to the proposed scheme are plotted at the 4th iteration. Equalizer (1) has coherent detection for first iteration; equalizer (2) has a 8-tap MMSE equalizer for first iteration.

3 stages for the iterative scheme to converge. The dash-dot curve marked with diamond represents the theoretical bound of this equalization algorithm derived previously in Section II-C. Clearly, the performance of this algorithm is far from its theoretical bound. Comparison between the solid curves with circle and the dashed curves with triangle indicates that the Proakis B channel is a harsher channel than the 5-tap static channel, however, they have the same performance lower bound, and consequently, a comparable performance should be expected for these two channels when turbo equalization is applied. Comparing Fig. 1 to Fig. 2, one can see that the static channels have a lower bound than the SUI-3 channel, thus holding a larger potential performance gain with turbo equalization.

The performance bound for the SUI-3 channel and the 5-tap static channel is further examined in Fig. 3, where we present both the analytical bound and the simulated bound of the equalizer. The latter is obtained by assuming the ISI is known, leading to perfect cancellation. Two cases are considered: i) perfect channel state information (CSI) and ii) the maximum likelihood channel estimation (CE). For the theoretical analysis, equation (15) in Section II-C is used to assess the performance bound for the static channel, while equation (17) in Section II-C is used for the SUI-3 channel. The parameters settings are $s^{2}=0.36, \sigma^{2}=0.175, \gamma_{1}=\mathrm{E}\left[\left|h_{1}\right|^{2}\right]=0.223, \gamma_{2}=\mathrm{E}\left[\left|h_{2}\right|^{2}\right]=0.07$ in equation (17), which are chosen according to [18] for the SUI-3 channel model. Fig. 3 shows that the theoretical analyses are in close agreement with the simulation results for both the SUI-3 and the static channel. Comparison between the simulation results with perfect CSI and the ones with CE indicate that the performance loss due to imperfect estimation is very small compared to the genie-aided case assuming perfect knowledge of CSI. 


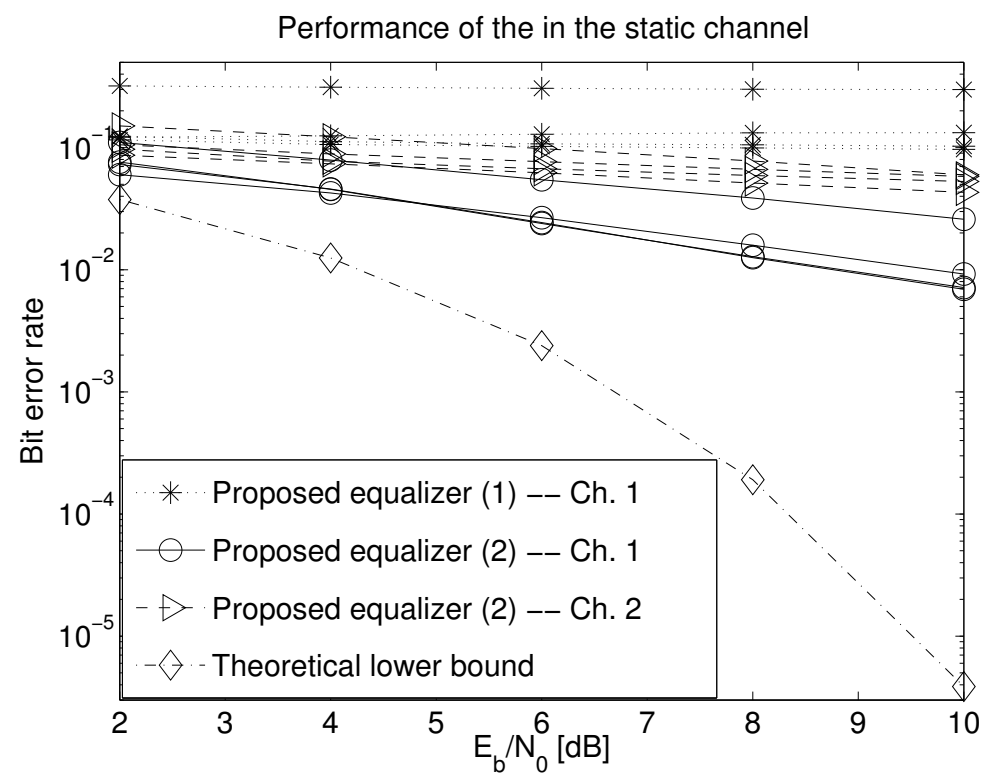

Fig. 2. Performance of the Jacobi algorithm for the static channels. For different implementations of the proposed equalizer, the topmost curve represents the first iteration, i.e., coherent detection (MMSE equalization) stage and the bottommost curve represents the 4th iteration. Channel 1 is the 5-tap static channel; channel 2 is the Proakis B channel.

It is obvious to see from Fig. 1 to Fig. 3 that the proposed algorithm is far from its performance bound. The rationale is that errors in the decision feedback significantly degrade performance and prevent the algorithm from reaching its theoretical potential. There are various ways of tackling this problem, e.g., using channel coding to reduce the feedback error probability, and iterative equalization and decoding to approach the performance bound. Combining these ideas leads to a new turbo equalization algorithm, which will be described in the following section.

\section{Extension of The Scheme to Coded Systems}

In order to reduce the error propagation and exploit the potential offered by the previously described equalization algorithm, we apply channel coding to the system, the baseband representation of which is depicted in Figure 4. The information sequence $\left\{b_{n}\right\}$ is convolutionally encoded into code bits $\left\{u_{n}\right\}$, which are subsequently interleaved and each block of two coded and interleaved bits $u_{n}^{\prime}[0], u_{n}^{\prime}[1]$ is mapped into one of the four QPSK symbols and transmitted over the ISI channel. The interleaver and deinterleaver are denoted as $\Pi$ and $\Pi^{-1}$, respectively, in Fig. 4 and Fig. 5. The QPSK symbol at time instant $n$ is denoted as $s_{n}=x_{n}+j y_{n}$, where $x_{n}, y_{n}=\frac{ \pm 1}{\sqrt{2}}$, and $\left|s_{n}\right|^{2}=1$. The received signal is basically the same as (1) except that the symbols $\left\{s_{n}\right\}$ are now formed by coded bits rather than information bits.

Here, we focus on the turbo equalization algorithm which combines equalizer and channel decoder in an iterative fashion. A soft-input, soft-output (SISO) equalizer is designed to exchange soft information with a SISO decoder. The existing techniques can be broadly classified into trellis (MAP) based and 


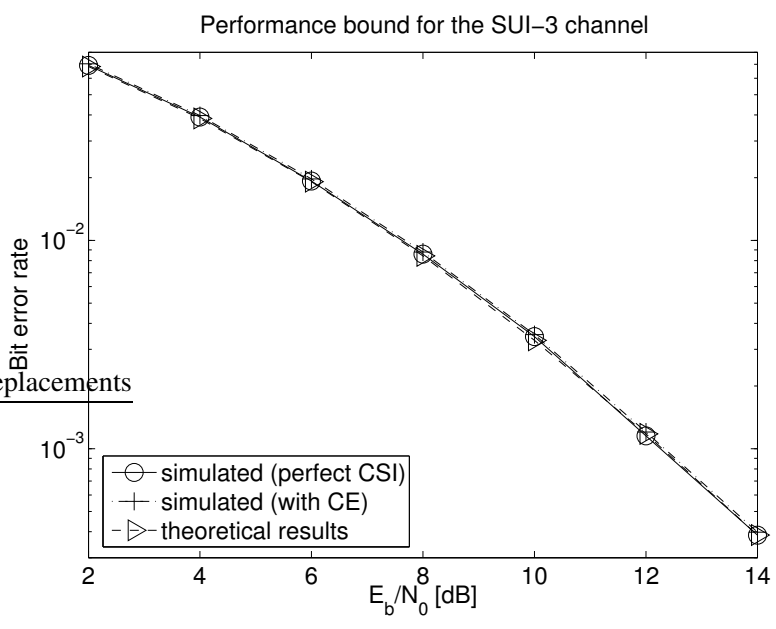

(a) Performance bound for the SUI-3 channel.

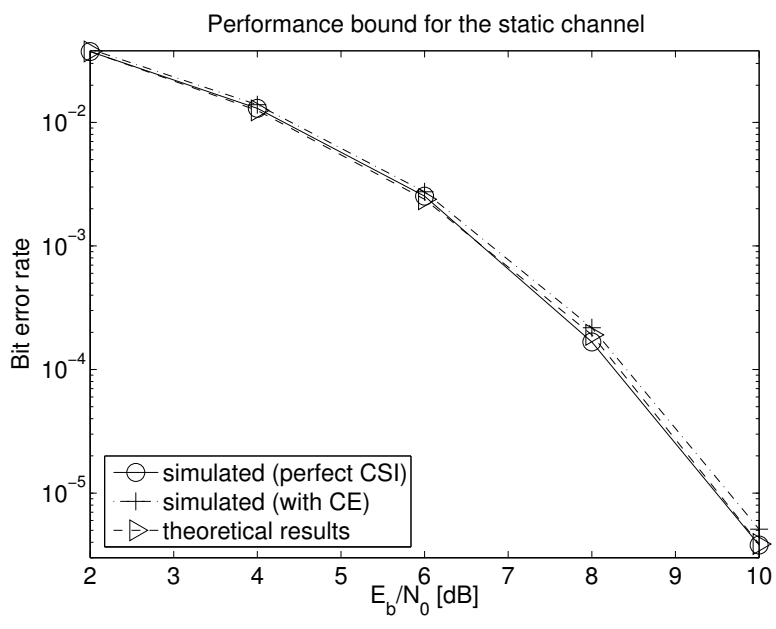

(b) Performance bound for the 5-tap static channel.

Fig. 3. Performance bound of the proposed equalizer: simulations vs. analyses, perfect CSI vs. CE.

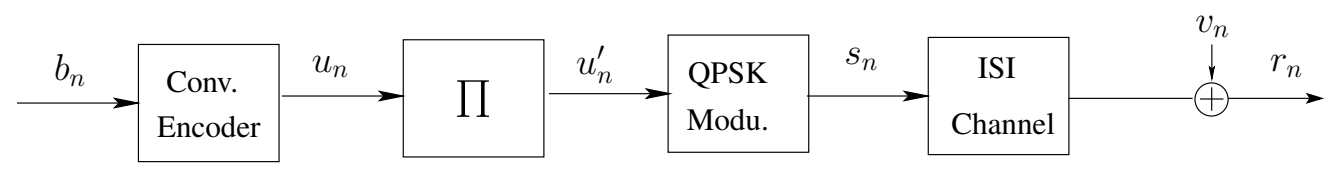

Fig. 4. Block diagram of a convolutionally coded QPSK system.

filter based approaches. For detailed descriptions of these turbo equalization algorithms, refer to [1]-[5]. Next, we introduce a new algorithm, which is an extension of the aforementioned equalization scheme.

\section{A. Turbo equalization algorithm}

The proposed turbo equalization algorithm is illustrated in Fig. 5. First, we use a training sequence to acquire a channel estimate $\hat{\mathbf{h}}$. In the meantime, a simple linear equalizer (LE) can be used to obtain an initial soft estimate of the transmitted symbols $\left\{\bar{s}_{n}=\bar{x}_{n}+j \bar{y}_{n}\right\}$. The channel estimate $\hat{\mathbf{h}}$ and symbol estimates $\left\{\bar{s}_{n}\right\}$ are passed to the equalizer (the SISO inner block shown in Fig. 5), which computes the loglikelihood ratio (LLR) value of $s_{n}$, denoted by $\lambda\left(s_{n}\right)=\lambda\left(x_{n}\right)+j \lambda\left(y_{n}\right)$. We use the notations $\lambda(\cdot ; I)$ and $\lambda(\cdot ; O)$ to denote the input and output ports of a SISO device. The LLR values of the symbols are mapped into LLR values of coded bits $\left\{\lambda\left(u_{n}^{\prime} ; O\right)\right\}$, which are deinterleaved to yield $\left\{\lambda\left(u_{n} ; I\right)\right\}$. For the QPSK modulated signals, the symbol to bit LLR mapping rule is simply $\lambda\left(u_{n}^{\prime}[0] ; O\right)=\lambda\left(x_{n}\right), \lambda\left(u_{n}^{\prime}[1] ; O\right)=$ $\lambda\left(y_{n}\right)$. Based on the soft input $\lambda\left(u_{n} ; I\right)$, a SISO outer channel decoder computes the LLR of each information bit $\lambda\left(b_{n} ; O\right)$ and each coded bit $\lambda\left(u_{n} ; O\right)$, where the former is used to make decisions on the transmitted information bit at the final iteration, and the latter is interleaved and passed through a bit-to-symbol converter (BSC) to derive a soft symbol estimate $\bar{s}_{n}=\bar{x}_{n}+j \bar{y}_{n}=\tanh \left[\lambda\left(u_{n}^{\prime}[0]\right) / 2\right] / \sqrt{2}+$ 


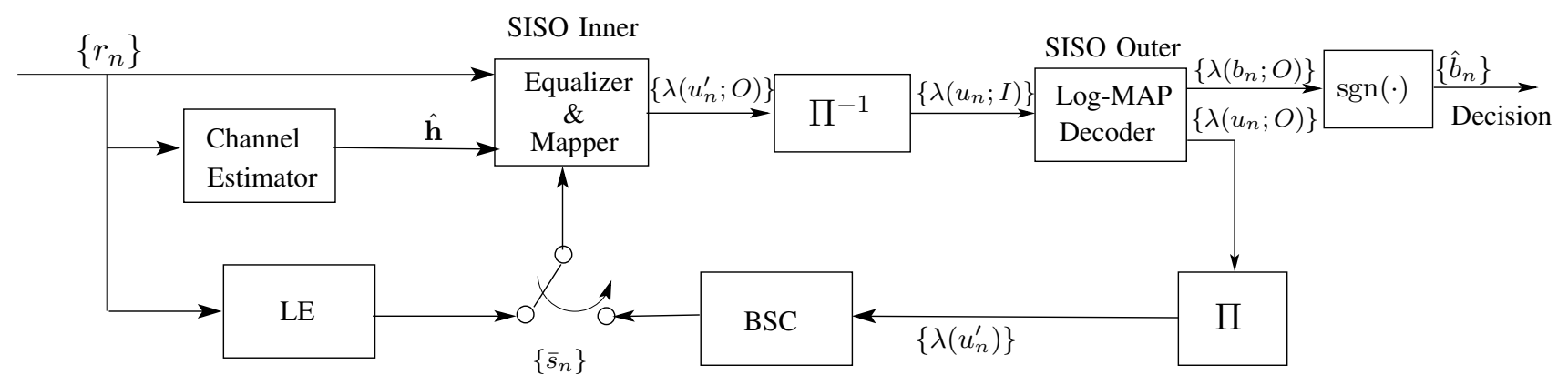

Fig. 5. Block diagram of the proposed turbo equalization scheme.

$j \tanh \left[\lambda\left(u_{n}^{\prime}[1]\right) / 2\right] / \sqrt{2}$, which is used for equalization at the next iteration. Several SISO algorithms can be used to compute the channel decoder outputs. For the purpose of this study, we consider the use of the Log-MAP algorithm [19]. We use the same equalization algorithm as described in Section II-B for the SISO inner block.

In the beginning of the iterative process, we can either assume no priori information $\left(\overline{\mathbf{s}}_{n}=\mathbf{0}\right.$ in this case), or use the soft symbol estimates derived in the pre-processing stage described previously. These two alternative solutions are compared in Section IV, and results show that pre-processing significantly improves the performance of the proposed scheme for some severe ISI channels. For the pre-processing, we can use a linear MMSE filter to obtain an initial estimate of the transmitted symbols (see Equ. (11) and Equ. (12) in Section II-B). Next, we explain how LLR values are derived based on the MMSE filter output so that the interference cancellation and iterative process can be carried out. It was shown in [20] that the MMSE filter output $z_{n}$ can be well approximated as a Gaussian random variable, i.e., $z_{n}=\mu s_{n}+\eta=\mu\left(x_{n}+j y_{n}\right)+\eta$ where $\eta \sim \mathcal{C N}\left(0, N_{\eta}\right)$. The real-valued parameters $\mu, N_{\eta}$ can be determined by taking expectation with respect to the interfering symbols and the channel noise

$$
\begin{aligned}
\mu & =\mathrm{E}\left\{z_{n} s_{n}^{*}\right\}=\mathbf{c}_{n}^{*} \mathrm{E}\left[\mathbf{r}_{n} s_{n}^{*}\right]=\mathbf{c}_{n}^{*} \mathbf{p}^{*}=\left(\mathbf{p} \mathbf{c}_{n}\right)^{*} ; \\
N_{\eta} & =\mathrm{E}\left[|\eta|^{2}\right]=\mathrm{E}\left[\left|z_{n}-\mu s_{n}\right|^{2}\right]=\mathrm{E}\left[\left(z_{n}-\mu s_{n}\right)\left(z_{n}^{*}-\mu s_{n}^{*}\right)\right] \\
& =\mathrm{E}\left\{\left|z_{n}\right|^{2}\right\}-\mu^{2}=\mu-\mu^{2} .
\end{aligned}
$$

The above equation holds since $z_{n}=\mathbf{c}_{n}^{*} \mathbf{r}_{n}$ and $\mathbf{c}_{n}=\mathbf{R}^{-1} \mathbf{p}^{*}$. Therefore,

$$
\mathrm{E}\left\{\left|z_{n}\right|^{2}\right\}=\mathrm{E}\left\{\mathbf{c}_{n}^{*} \mathbf{r}_{n} \mathbf{r}_{n}^{*} \mathbf{c}\right\}=\mathbf{c}_{n}^{*} \mathbf{R} \mathbf{c}_{n}=\mathbf{p} \mathbf{R}^{-1} \mathbf{R} \mathbf{c}_{n}=\mathbf{p} \mathbf{c}_{n}=\mu
$$

After computing the values of $\mu$ and $N_{\eta}$, the conditional PDF of the equalizer output can be obtained as $f\left(z_{n} \mid s_{m}\right)=\frac{1}{\pi N_{\eta}} \exp \left(-\frac{\left|z_{n}-\mu s_{m}\right|^{2}}{N_{\eta}}\right)$, and the LLR value of $x_{n}$ can thus be computed as

$$
\begin{aligned}
\lambda\left(x_{n}\right) & =\ln \frac{f\left(z_{n} \mid x_{n}=0\right)}{f\left(z_{n} \mid x_{n}=1\right)}=\ln \frac{f\left(z_{n} \mid s_{0}\right)+f\left(z_{n} \mid s_{3}\right)}{f\left(z_{n} \mid s_{1}\right)+f\left(z_{n} \mid s_{2}\right)} \approx \ln \frac{\exp \left(-\left|z_{n}-\mu s_{+}\right|^{2} / N_{\eta}\right)}{\exp \left(-\left|z_{n}-\mu s_{-}\right|^{2} / N_{\eta}\right)} \\
& =\frac{1}{N_{\eta}}\left\{\left|z_{n}-\mu s_{-}\right|^{2}-\left|z_{n}-\mu s_{+}\right|^{2}\right\}=\frac{2}{1-\mu} \operatorname{Re}\left\{s_{+}^{*} z_{n}-s_{-}^{*} z_{n}\right\},
\end{aligned}
$$


where $s_{+}$denotes the QPSK symbol corresponding to $\max \left\{f\left(z_{n} \mid s_{0}\right), f\left(z_{n} \mid s_{3}\right)\right\}$, and $s_{-}$denotes the QPSK symbol corresponding to $\max \left\{f\left(z_{n} \mid s_{1}\right), f\left(z_{n} \mid s_{2}\right)\right\}$ since the real part of the symbols $s_{0}, s_{3}$ corresponds to 0 , and the real part of the symbols $s_{1}, s_{2}$ corresponds to 1 . The dual maxima rule [21] is used in (19) utilizing the fact that one term usually dominates each sum. Similarly,

$$
\lambda\left(y_{n}\right)=\ln \frac{f\left(z_{n} \mid s_{0}\right)+f\left(z_{n} \mid s_{1}\right)}{f\left(z_{n} \mid s_{2}\right)+f\left(z_{n} \mid s_{3}\right)} \approx \frac{2}{1-\mu} \operatorname{Re}\left\{s_{+}^{*} z_{n}-s_{-}^{*} z_{n}\right\},
$$

where $s_{+}$denotes the QPSK symbol corresponding to $\max \left\{f\left(z_{n} \mid s_{0}\right), f\left(z_{n} \mid s_{1}\right)\right\}$, and $s_{-}$denotes the QPSK symbol corresponding to $\max \left\{f\left(z_{n} \mid s_{2}\right), f\left(z_{n} \mid s_{3}\right)\right\}$ since the imaginary part of the symbols $s_{0}, s_{1}$ corresponds to 0 , and the imaginary part of the symbols $s_{2}, s_{3}$ corresponds to 1 . Finally, the soft symbol estimate can be derived based on the derived LLRs, i.e., $\bar{s}_{n}=\bar{x}_{n}+j \bar{y}_{n}=\tanh \left[\lambda\left(x_{n}\right) / 2\right] / \sqrt{2}+$ $j \tanh \left[\lambda\left(y_{n}\right) / 2\right] / \sqrt{2}$. In the subsequent stages, $\bar{s}_{n}$ is derived based on the output of the Log-MAP decoder.

The LLR value at the output of the SISO inner block can be derived similarly based on (9). For simplicity, we assume the filter output is ISI-free, the decision statistic takes the form of (14), from which the LLR value can be easily obtained as shown previously. Due to the ISI-free assumption, this scheme is sub-optimum during the initial stages of turbo equalization, but will approach optimality when the ISI is effectively canceled as the iterative process proceeds.

\section{B. Performance bound for the turbo equalization}

Like we did in Section II-C for the uncoded case, we analyze the performance bound of the proposed turbo equalization scheme in order to gain an insight into its asymptotic performance. Assume perfect cancellation, the decision statistics in (9) becomes

$$
z_{n}=s_{n}+\frac{1}{\mathcal{P}} \sum_{l=0}^{L-1} h_{l}^{*} v_{n-l}=s_{n}+w_{n}=x_{n}+w_{I}+j\left(y_{n}+w_{Q}\right),
$$

where $w_{n}=w_{I}+j w_{Q} \sim \mathcal{C N}\left(0, N_{w}\right), N_{w}=\sum_{l=0}^{L-1}\left|h_{l}\right|^{2} N_{0} / \mathcal{P}^{2}=N_{0} / \mathcal{P}$, and $w_{I}, w_{Q}$ are independent zero mean Gaussian random variables with variance $\frac{N_{0}}{2 \mathcal{P}}$. Assume all zero bits are transmitted, then $s_{n}=\sqrt{E_{b}}+j \sqrt{E_{b}}, x_{n}=y_{n}=\sqrt{E_{b}}$. In the derivation of the theoretical lower bound, we use the facts that the Max-Log-MAP algorithm provides exactly the same hard decisions as the Viterbi algorithm [19], and the Max-Log-MAP is an approximation of the Log-MAP, i.e., it does not include a correction term. Since the modulation/demodulation of a QPSK system is equivalent to two independent (phase-quadrature) BPSK systems [10], this coded QPSK system has equivalent performance with a coded BPSK system with $r_{i}=\sqrt{E_{b}} c_{i}+u_{i}$ at the input of the Viterbi decoder, where $c_{i}= \pm 1$, with +1 corresponding to the binary digit 0 and -1 corresponding to 1 . The noise term $u_{i}$ has the same variance as $w_{I}$ and $w_{Q}$, i.e., $u_{i} \sim \mathcal{N}\left(0, N_{u}\right), N_{u}=\frac{N_{0}}{2 \mathcal{P}}$. The conditional PDF is thus $p\left(r_{i} \mid c_{i}\right)=\frac{1}{\pi N_{u}} \exp \left(-\frac{\left|r_{i}-\sqrt{E_{b}} c_{i}\right|^{2}}{N_{u}}\right)$. Assuming the maximum likelihood decoding, after neglecting the common terms, the metrics corresponding to the all-zero path and the first error event path can be expressed as

$$
C M^{(0)}=\sum_{l=1}^{d}(+1)\left(\sqrt{E_{b}}+u_{l}\right) ; \quad C M^{(1)}=\sum_{l=1}^{d}(-1)\left(\sqrt{E_{b}}+u_{l}\right),
$$


where the index $l$ runs over the set of $d$ bits since the coded bits in the two paths are identical except in $d$ positions, and the common terms due to the identical bits are left out in the above metrics. The pairwise error probability $P_{2}(d)$, which is defined as the probability of decoding in favor of a codeword with weight $d$ when all-zero codeword is transmitted, is computed as the probability that the error path has better metric than the all-zero path, i.e.,

$$
\begin{aligned}
P_{2}(d) & =P_{r}\left(C M^{(1)}>C M^{(0)}\right)=P_{r}\left[\sum_{l=1}^{d}(-1)\left(\sqrt{E_{b}}+u_{l}\right)>\sum_{l=1}^{d}(+1)\left(\sqrt{E_{b}}+u_{l}\right)\right] \\
& =P_{r}\left[2 \sum_{l}^{d} u_{l}<-2 d \sqrt{E_{b}}\right]=P_{r}\left[\eta<-d \sqrt{E_{b}}\right]=Q\left(\frac{d \sqrt{E_{b}}}{\sqrt{\frac{d N_{0}}{2 \mathcal{P}}}}\right)=Q\left(\sqrt{\frac{2 d \mathcal{P} E_{b}}{N_{0}}}\right),
\end{aligned}
$$

where $\eta=\sum_{l=1}^{d} u_{l}$, and $N_{\eta}=d N_{u}=\frac{d N_{0}}{2 \mathcal{P}}$. The bit error probability $P_{b}$ is upper bounded by [10]

$$
P_{b} \leq \sum_{d=d_{\text {free }}}^{\infty} c_{d} P_{2}(d)=\sum_{d=d_{\text {free }}}^{\infty} c_{d} Q\left(\sqrt{\frac{2 E_{b} d \mathcal{P}}{N_{0}}}\right) \approx \sum_{d=d_{\text {free }}}^{N_{t}} c_{d} Q\left(\sqrt{\frac{2 E_{b} d \mathcal{P}}{N_{0}}}\right)
$$

where $d_{\text {free }}$ is the free distance of the code, and $c_{d}$ is the sum of the information weights of all error paths with weight $d$, which can be computed from the transfer function of the code. However, $c_{d}$ is also tabulated, e.g., in [23] for most good codes of practical interest, including the one we have used in our simulations. In equation (20), $N_{t}$ denotes the truncation length. The pairwise error probability $P_{2}(d)$ is rapidly decreasing with $d$. Hence, for a sufficient high value of $d>N_{t}$, the terms $c_{d} P_{2}(d)$ will be negligible, and we can truncate the sum without compromising the bound. The above equation works for static channels. For non-static channels, we have to average the error probability over the distributions of channel gains of different paths as we did in the uncoded case.

\section{Numerical Results For Turbo Equalization}

The proposed scheme is evaluated and compared numerically with some existing algorithms in this section. In particular, we make a comparison with the MMSE filter based turbo equalization proposed by Tüchler, et. al. in [3], and the adaptive turbo equalization introduced by Laot, et. al. in [5] as well as some frequency domain solutions, such as OFDM and SC-FDE. Note that the turbo equalization scheme presented by Wang and Poor in [2] is identical to Tüchlers scheme in a single-user case. Two approximate implementations of Tüchler's scheme was given in [3]. They have lower complexity than the original algorithm, but also lead to some degree of performance loss. Here, we only use its original implementation for performance comparison. The modified versions of those existing schemes tailored for the QPSK modulated system under investigation are used for comparison purpose. In the simulations, we employ a rate 1/3 Maximum Free Distance convolutional code [10] with constraint length 5 and generator polynomials $(25,33,37)_{8}$. During each Monte-Carlo run, the block size is set to 1360 information bits followed by 4 tails bits to terminate the trellis, which corresponds to $1364 \times 3=4092$ coded bits. They are interleaved by an random interleaver (unless otherwise stated) and transmitted over an ISI 
channel. The noise variance $N_{0}$ and path delays are assumed to be known to the receiver. For the initial equalization, we use a 8-tap linear MMSE equalizer, and 200 pilot symbols are used for training the equalizer coefficients. In the meantime, the modified maximum likelihood algorithm presented in [17] is used for channel estimation during the training period. The channel estimates are averaged over many estimated samples to reduce the noise effect. To study the behavior of each algorithm, the number of iterations is usually set to 4 , since it is observed that almost all the algorithms would converge after 3 or 4 iterations.

Both time-varying (SUI-3) and the static channels (the 5-tap static channel and Proakis B channel) are used in our simulations. The performance comparison between the proposed scheme and some existing turbo equalization schemes is given in Fig. 6. For Laot's adaptive algorithm [5], the step size $\mu$ is set to 0.006 during the training period and 0.002 during the tracking period. 200 symbols are used for training the equalizer coefficients. Note that the performance of this adaptive scheme can be improved by transmitting more pilots at the expense of larger overhead and a decrease in the system spectrum efficiency. Both random interleaver and a $66 \times 62$ block interleaver are tested for the 5 -tap static channel and Proakis B channel. It can be seen from Fig. 6.a)-c) that when random interleaver is used, the proposed algorithm yields almost the identical results to the Tüchler's MMSE scheme, while achieves a gain of up to $1 \mathrm{~dB}$ compared to Laot's adaptive scheme after the system reaches convergence.

Fig. 6.b) and Fig. 6.c) also show that interleaver is crucial for the performance of the turbo equalizers. However, it has different impact for different equalizers and in different channel conditions. For example, the use of random interleaver instead of block interleaver significantly improves the performance of the Laot's scheme in both channels, however, its impact on the other two schemes are not as significant for channels with mild ISI condition, such as the 5-tap static channel; whereas for a harsher channel such as Proakis B channel, the replacement of block interleaver with random interleaver significantly improves the performance for all the discussed schemes.

The soft information used by the proposed equalizer is the full a posteriori information at the output of the channel decoder. The same arrangement has been made, e.g., in [3]. Although it is a common practice to use the extrinsic information in iterative detection/decoding, no noticeable distinction in terms of performance between these two different arrangements has been observed for the proposed turbo equalizer as shown by Fig. 6.d). The same comments can be found in [25]. In some situations, e.g., with the MIMO iterative detector proposed in [26], iterative detectors with a posteriori information as feedback even perform better than the ones with extrinsic feedback. In Fig. 6.d), we also compare the proposed scheme with Tüchler's approximate solution I. Upon reaching convergence at the 4th iteration, the former outperforms the latter by more than $1 \mathrm{~dB}$ at the target $\mathrm{BER}=10^{-4}$.

We know from the above experiments that when the condition of perfect cancellation is satisfied or approached, the proposed scheme does not incur a performance penalty compared to the MMSE scheme. However, when the condition of perfect cancellation is not satisfied, the performance of the 


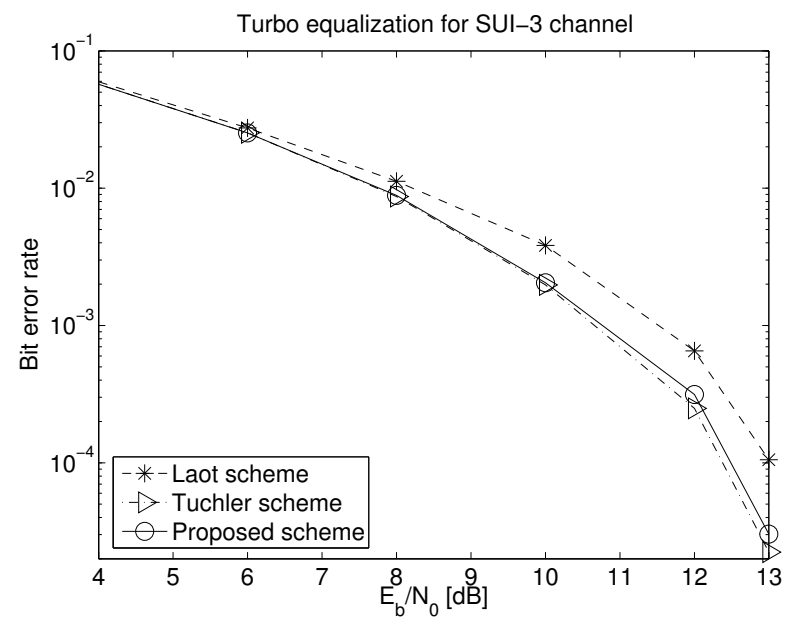

(a) For the SUI-3 channel (R. int.).

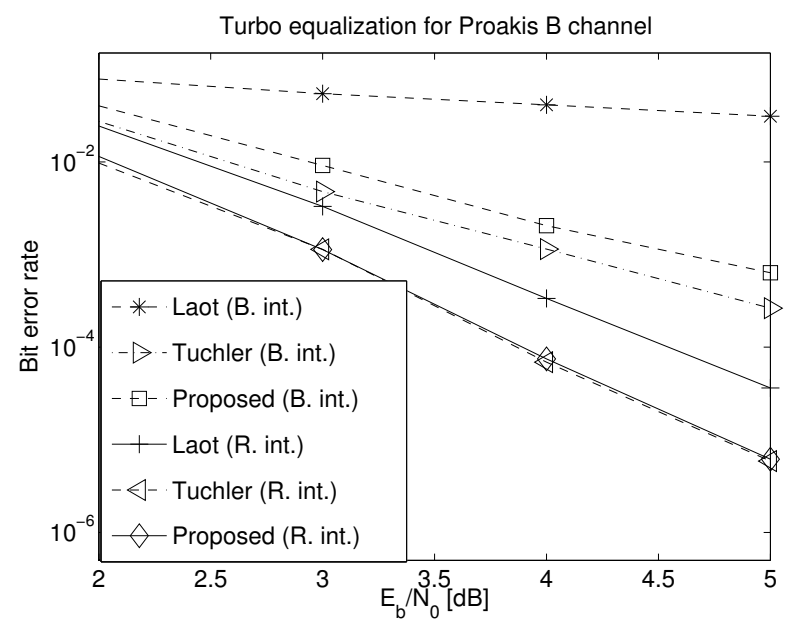

(c) For Proakis B channel.

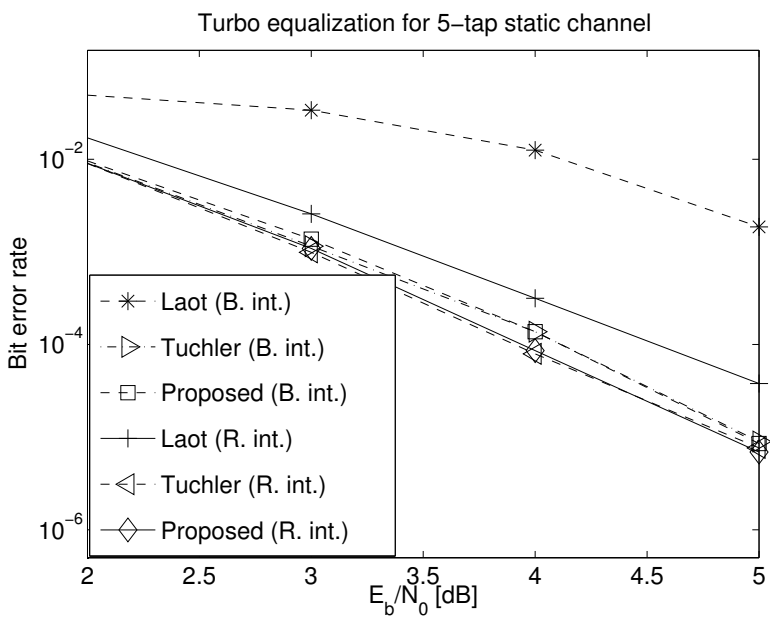

(b) For the 5-tap static channel.

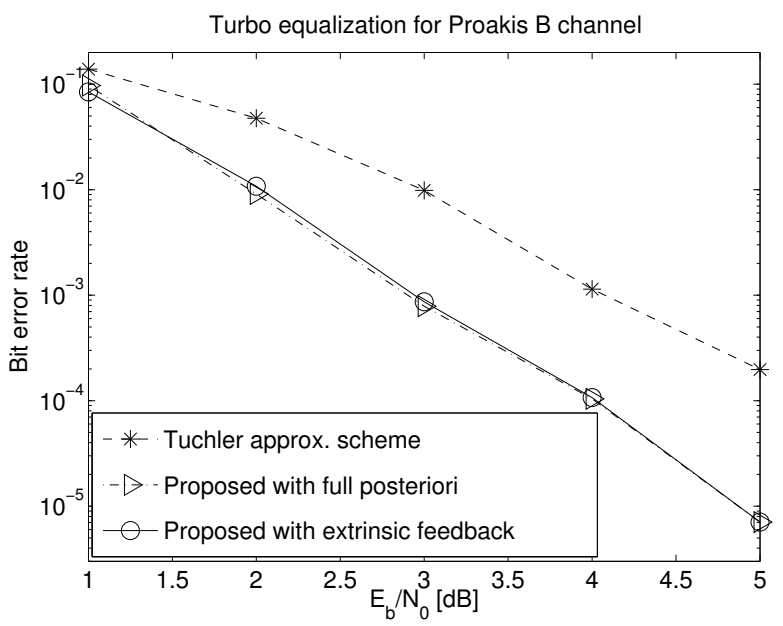

(d) For Proakis B channel (R. int.).

Fig. 6. Comparison of different turbo equalization algorithms after reaching convergence. All the curves represent the 4th iteration turbo equalization. B. int. stands for block interleaving; R. int. stands for random interleaving. Proposed scheme (1) refers to the one assuming no priori information at the first iteration; proposed scheme (2) refers to the one using LE preprocessing at the first iteration.

proposed turbo equalization becomes suboptimum. One example is shown in Fig. 6.c) where we see that the Tüchler's scheme performs better than the proposed scheme with block interleaver for the Proakis B channel. To further investigate the performance loss under such circumstances, we apply different schemes to another static channel with more frequency-selectivity (Proakis D channel) having impulse response $h[n]=0.1275 \delta[n]+0.450 \delta[n-1]+0.750 \delta[n-2]+0.450 \delta[n-3]+0.1275 \delta[n-4]$. Fig. 7 shows that even with random interleaver, the proposed scheme is far from the performance bound, ISI-free transmission cannot be fulfilled for this harsh channel. Tüchler's MMSE scheme has the best 


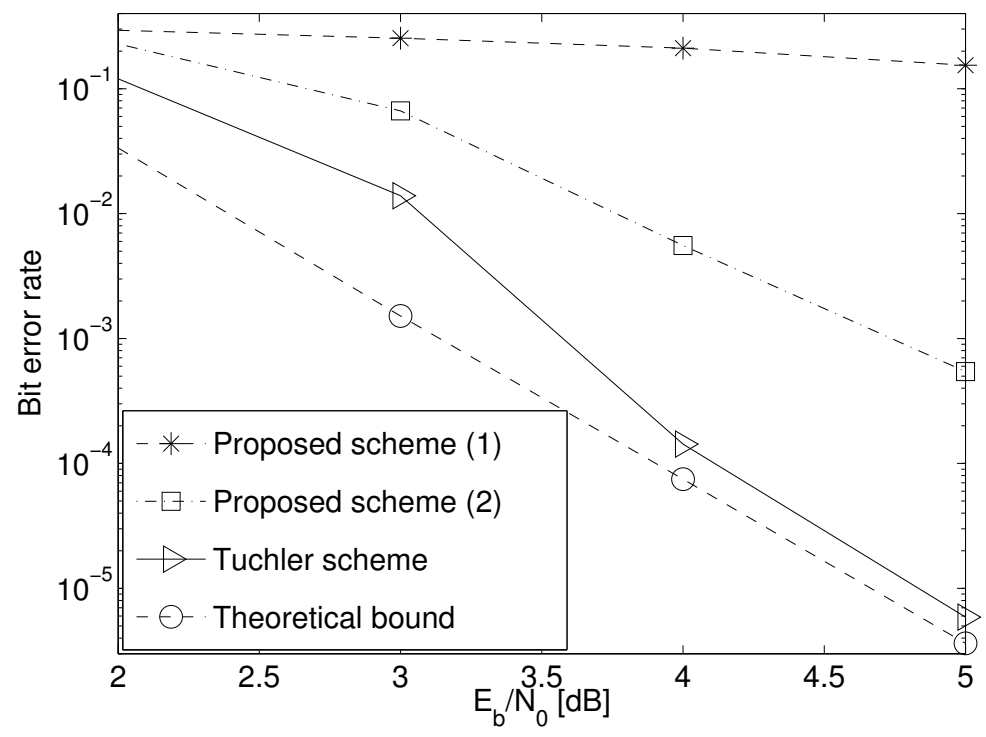

Fig. 7. Comparison of different turbo equalization algorithms in Proakis D channel. Random interleaving is used. The curves for the proposed and the Tüchler's schemes represent the 5th iteration turbo equalization.

performance, and approaches the performance bound at high SNR. The theoretical bound is obtained by (20), the truncation length is chosen to be $N_{t}=30$. We observe a gain of $1.3 \mathrm{~dB}$ at $\mathrm{BER}=10^{-3}$ by performing MMSE filtering in this case. Two variants of the proposed scheme are tested. Apparently, the one with LE pre-processing performs much better than the one assuming no priori information at the first stage. We also noticed that the performance gap between these two variants of the scheme is smaller when the channel ISI condition is less severe, e.g., for the 5-tap static channel and the Proakis B channel. The plots are omitted here to conserve space.

The Jacobi algorithm as described in this paper is only one of the several iterative schemes for solving linear system equations without matrix inversion. Other methods include Jacobi over-relaxation (JOR), serial Guass-Seidel (GS), conjugate gradient, etc.. In the JOR method, the symbol detected at the current iteration consists of the solution produced by the Jacobi algorithm multiplied by an over relaxation factor $\alpha$ plus the previous solution multiplied by $1-\alpha$. The over relaxation factor $\alpha$ is chosen to adance the solution more quickly than would be the case with the standard Jacobi iterative method. In the serial GS method, soft estimates of past symbols can be updated at the current iteration based on the output of the interference canceller, while future symbols are still estimated based on soft information from the previous iteration. JOR and GS methods will be considered in our future work to achieve faster convergence and improve the performance of the turbo equalizer over severely ISI-distorted channels.

In Fig. 8, the proposed turbo equalization scheme is compared with the performance bound for the two static channels. The simulated bound is obtained by assuming perfect knowledge of the transmitted symbols and channel state information (CSI) in the simulations, leading to perfect cancellation. The 


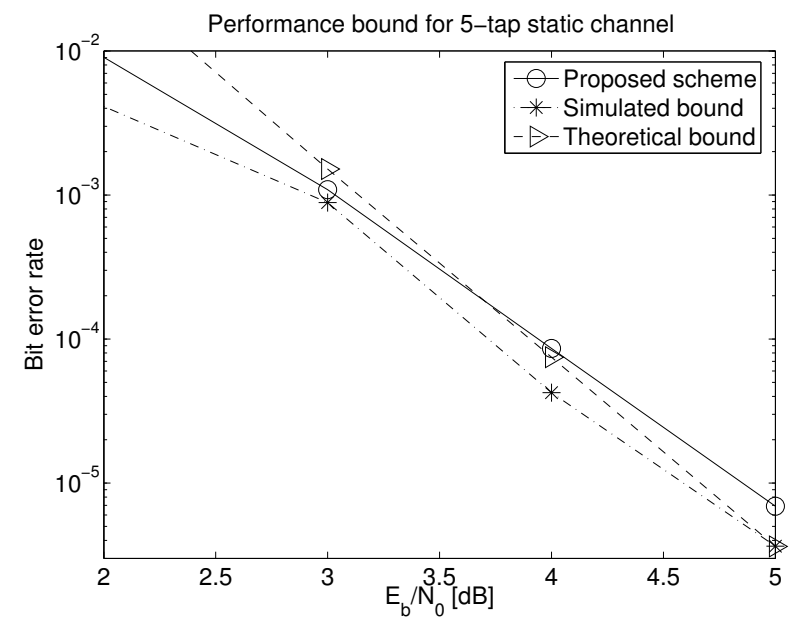

(a) For the 5-tap static channel.

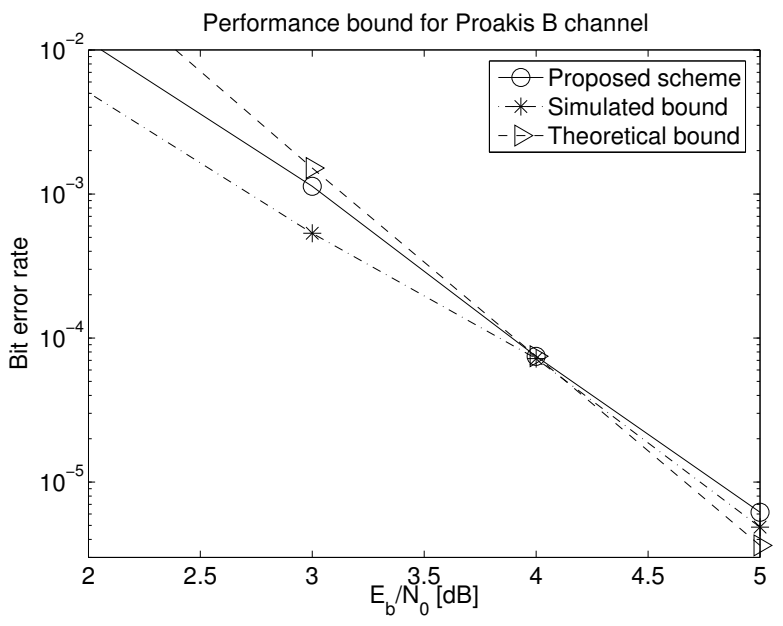

(b) For Proakis B channel.

Fig. 8. Comparison between the proposed turbo equalization scheme and its performance lower bound. The curve for the proposed scheme represents the 4th stage turbo equalization.

theoretical bound is given by (20) in Section III-B. The plot shows fairly close agreement between the theoretical bound and the simulated bound as long as the BER is below $10^{-3}$, which means that the derived theoretical bound provides good insight into the asymptotic behavior of the proposed scheme. As shown in [24], the union bound expressed by (20) gives a fairly good estimate of the bit error rate performance at BERs below $10^{-3}$. Therefore, it is not surprising to see that the turbo equalizer lies below the analytical bound when the BER is above $10^{-3}$. The same can be observed from Figs. 6, 7, 8 in [24]. Fig. 8 also shows that the performance of the proposed scheme is very close to the performance bound after it reaches convergence. This indicates that the effect of ISI can be effectively removed by the proposed turbo equalization algorithm, and ISI-free transmission is approached for these two channels. The potential for performance improvement predicted in Fig. 2 and Fig. 3 is realized by extending the algorithm to coded systems and by applying the turbo processing principle.

The proposed scheme is compared with OFDM and SC-FDE in Fig. 9, where the performance of different schemes is shown for systems employing the convolutional codes with rate $1 / 3$ and rate $1 / 2$, respectively. In the former case, the code generator polynomials is chosen to be $(25,33,37)_{8}$. The data frame size is set to 1360 information bits followed by 4 tails bits to terminate the trellis. Four zeros are appended at the end of the bit sequence to make the total number of transmitted bits equal to $2^{12}$. In the latter case, the code generator polynomials is chosen to be $(23,35)_{8}$. The frame size is set to 2044 information bits followed by 4 tails bits. For simplicity, we assume perfect channel estimation. For the OFDM/SC-FDE system, each frame of data contains 4096 coded bits (2048 QPSK symbols), and is divided into 8 OFDM blocks. The number of sub-carriers is $N=256$, and the length of cyclic prefix 


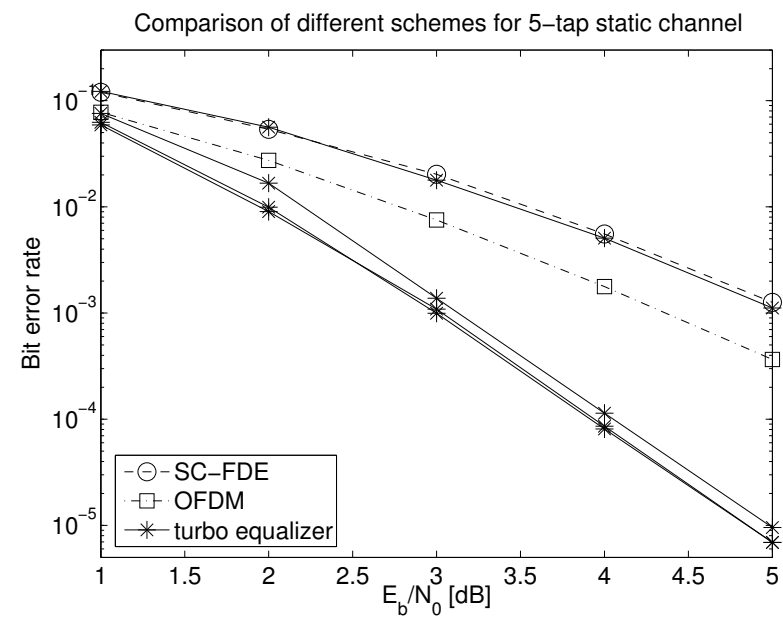

(a) For the 5-tap static channel (1/3 code).

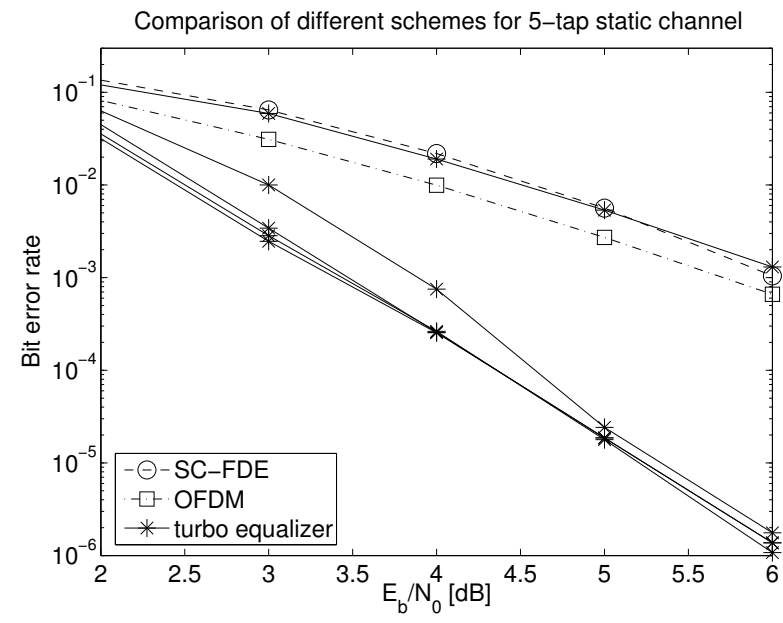

(c) For the 5-tap static channel (1/2 code).

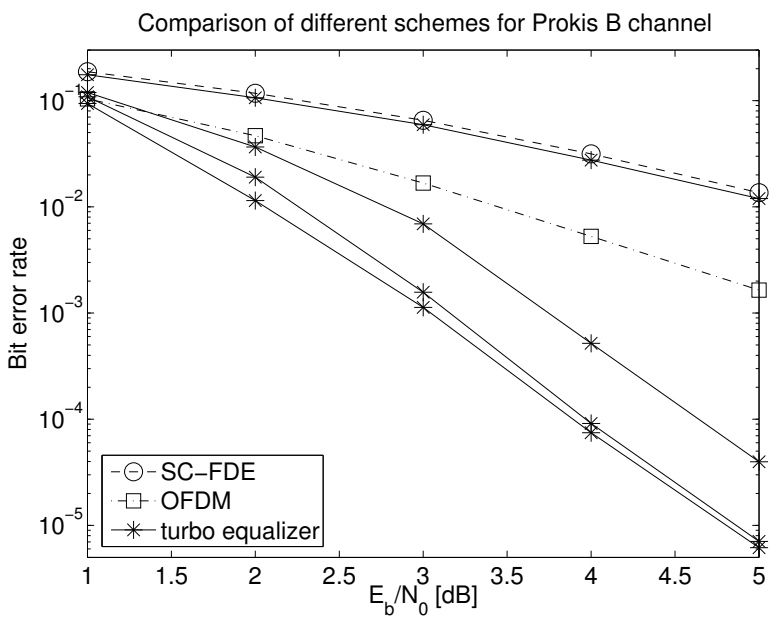

(b) For Proakis B channel (1/3 code).

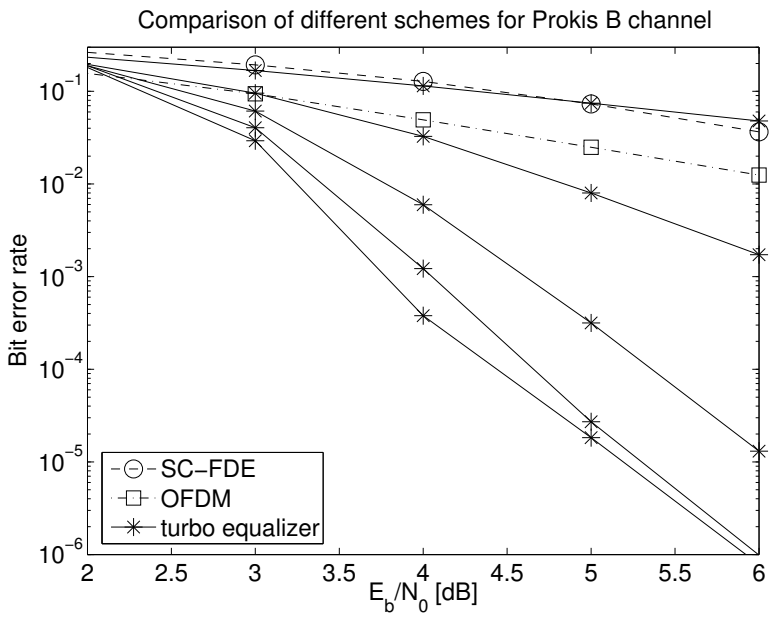

(d) For Proakis B channel (1/2 code).

Fig. 9. Comparison between time and frequency domain schemes. For the proposed turbo equalizer, the topmost curve represent the first stage equalization (after LE pre-processing) and Log-MAP decoding, the bottom curve represents the 4th stage turbo equalization (5th stage turbo equalization for Proakis B channel with 1/2 code).

is 8. One can observe from Fig. 9 that the proposed turbo equalizer outperforms the SC-FDE/OFDM scheme at the 2 nd iteration. Upon reaching convergence, the proposed turbo equalizer performs much better than OFDM/SC-FDE, e.g., it outperforms the OFDM scheme by 1.5 (2.5) $\mathrm{dB}$ at $\mathrm{BER}=10^{-3}$ with rate $1 / 3(1 / 2)$ code for the 5-tap static channel. We also see from the figure that OFDM performs better than SC-FDE, however, their performance gap is smaller in the system with rate $1 / 2$ code than in the system with rate $1 / 3$ code. This concurs with the results given in [27], where it shows that as the code rate increases, e.g., to $R_{c}=2 / 3$, SC-FDE begins to outperform OFDM since a system with high rate code is close to an uncoded system for which SC-FDE outperforms OFDM [8]. 
Fig. 9 also shows the convergence property of the proposed scheme. In most cases, it takes only 3 or 4 stages for the algorithm to converge. Compared to the initial stage with one time equalization and Log-MAP decoding without feedback, the subsequent turbo equalization stages achieve much better performance, and most significant gains are obtained at first 3 stages. The gain by applying turbo equalization increases as SNR increases.

The convergence behavior of the proposed turbo equalizer is examined at $E_{b} / N_{0}=3 \mathrm{~dB}$ for the 5-tap static channel in Fig. 10 using the extrinsic information transfer (EXIT) chart which traces the evolution of the mutual information $I_{i}^{E} / I_{o}^{E} \in[0,1]$ between input/output LLR and $s_{n}$ for equalization; and the mutual information $I_{i}^{D} / I_{o}^{D}$ between input/output LLR and $u_{n}$ for decoding. The priori information $L_{i}$ is usually modeled as Gaussian random variable with PDF

$$
f_{L_{i}}(l \mid x)=\frac{1}{\sqrt{2 \pi} \sigma_{i}} \exp \left(-\frac{\left(l-x \sigma_{i}^{2} / 2\right)^{2}}{2 \sigma_{i}^{2}}\right)
$$

i.e., $L_{i} \sim \mathcal{N}\left(\frac{1}{2} \sigma_{i}^{2}, \sigma_{i}^{2}\right)$. The mutual information $I_{i}$ is essentially a function of the single parameter $\sigma_{i}$ [28]. For a range of values of $\sigma_{i}$, a histogram of the output LLRs $P_{L_{o}}(l \mid x)$ is generated based on extensive simulations. The PDF of the random variable $L_{o}$ of output LLRs is then estimated for the equalizer and decoder from this histogram. Mutual information $I_{i}$ and $I_{O}$ can then be computed numerically as [29]

$$
\begin{aligned}
I_{i} & =\frac{1}{2} \sum_{x \in\{ \pm 1\}} \int_{-\infty}^{\infty} f_{L_{i}}(l \mid x) \log _{2} \frac{2 f_{L_{i}}(l \mid x)}{f_{L_{i}}(l \mid+1)+f_{L_{i}}(l \mid-1)} d l \\
I_{o} & =\frac{1}{2} \sum_{x \in\{ \pm 1\}} \sum_{l} \log _{2} \frac{2 P_{L_{o}}(l \mid x)}{P_{L_{o}}(l \mid+1)+P_{L_{o}}(l \mid-1)} .
\end{aligned}
$$

Refer to [28] for detailed discussion of this analysis method and [3], [30] for its application in turbo equalization.

It should be noted that in our case, $I_{i}^{E}>0$ at the beginning of the iterative process. This is due to the initial LE (see the lower left corner in Fig. 5), therefore, the a priori information is not zero when the turbo equalization starts. The output LLR of the equalizer $I_{o}^{E}$ is forwarded to the decoder as input, i.e., $I_{i}^{D}=I_{o}^{E}$; the output LLR of the decoder $I_{o}^{D}$ is fed back to the equalizer, i.e., $I_{i}^{E}=I_{o}^{D}$, and so on. Note that the interleaving/deinterleaving does not change mutual information. As indicated by the equalizer transfer curves in Fig. 10, the output LLR $I_{o}^{E}$ becomes more reliable (its value increases) as the input LLR $I_{i}^{E}$ becomes more reliable in the equalizer. The iterative equalization and decoding process is depicted by a staircase trace between the transfer curves of the equalizer and decoder. The trace for the proposed scheme shows that only 3 stages of equalization/decoding are needed for the system to converge (reach the maximum $I_{o}^{D}$ ). The majority of the gain is obtained at the second iteration beyond which the improvement becomes unnoticeable. This is in close agreement with the BER results shown in Fig. 9.a). The figure also shows that given perfect a priori information at the input of the equalizer, i.e., when $I_{i}^{E}=1$, the value of $I_{o}^{E}$ produced by the proposed scheme is approximately the same as (higher than) the one produced by the Tüchler's (Laot's) scheme. This coincides with the results shown 


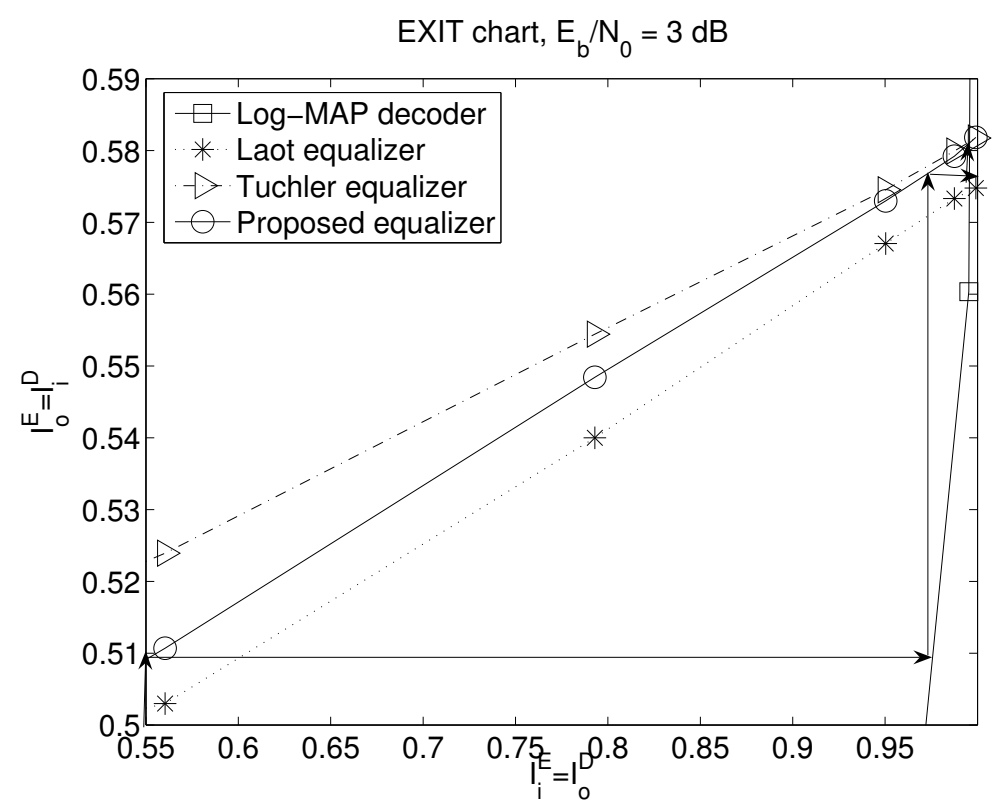

Fig. 10. EXIT chart of the turbo equalization for the 5-tap static channel at $E_{b} / N_{0}=3 \mathrm{~dB}$. In the plot, $\rightarrow$ represents the decoding process and $\uparrow$ represents the equalization process.

TABLE I

COMPARISON OF COMPLEXITY FOR THE ALGORITHMS CONSIDERED.

\begin{tabular}{|c|c|c|c|}
\hline \hline operations & multiplication & division & addition/subtraction \\
\hline Proposed scheme & $L^{2}+6$ & 2 & $L^{2}+1$ \\
\hline Tüchler's scheme & $8 L^{3}-4 L^{2}+3 L+8$ & $2 L^{2}+2$ & $8 L^{3}-3 L^{2}+4 L$ \\
\hline Tüchler approx. & $2 L^{2}+2 L+6$ & 2 & $2 L^{2}+2 L+1$ \\
\hline Laot's scheme & $6 L+4$ & 4 & $6 L+4$ \\
\hline
\end{tabular}

in Fig. 6.b), where we see that the proposed scheme yields comparable (superior) BER performance to the Tüchler's (Laot's) scheme upon reaching the convergence.

Table I shows the number of complex multiplications, divisions, and additions/subtractions required for the estimation of each QPSK symbol at each iteration for the turbo equalization schemes considered, where $L$ is the number of channel taps. The figures for the Tüchler's and Laot's schemes are based on their modified versions for QPSK modulation. One can see from the table that Laot's scheme has the lowest complexity, which is linear with $L$. However, it has the worst performance as one can see from the numerical results presented earlier. By avoiding matrix inversion, the proposed scheme reduces the complexity from $O\left(L^{3}\right)$ to $O\left(L^{2}\right)$ compared to the original Tüchler's scheme. For example, the original Tüchler's scheme requires $8 L^{3}-4 L^{2}+3 L+8$ and $8 L^{3}-3 L^{2}+4 L$ complex multiplications and additions/subtractions, respectively, to estimate one QPSK symbol at each iteration; whereas the proposed scheme reduce the figures to $L^{2}+6$ and $L^{2}+1$. It also has lower complexity than the approximate 
implementation of the Tüchler's scheme. Furthermore, its complexity is comparable to Laot's scheme when the delay spread is short, i.e., for small values of $L$.

OFDM and SC-FDE have essentially the same computational complexity, both require $\operatorname{Nlog}_{2} N+N$ complex multiplications for a FFT block with length $N$ [9]. Therefore, their complexity does not increase with $L$. However, considering the fact that channel dispersion grows linearly with the data rate, $N$ should be increased when data transmission rate goes higher in order to minimize the fraction of overhead due to the insertion of cyclic prefix. It is generally believed that time-domain equalization cannot cope with severe dispersive channels in high data rate broadband wireless transmission systems where ISI spans over one hundred symbols, and OFDM/SC-FDE is a more feasible solution under such circumstances. By comparison, OFDM/SC-FDE represents a more conservative solution, is not necessarily optimum, but guarantees operation in most environments [31]; whereas the turbo equalization scheme achieves better performance at the cost of higher computational complexity incurred by the iterative process.

\section{Conclusions}

In this paper, we first introduced a new approach to equalization based on the Jacobi algorithm. However, the results show that the performance of this algorithm is far from its theoretical potential in an uncoded system, due to the fact that errors in the decision feedback significantly degrade its performance. This suggests the use of channel coding to reduce the effect of feedback propagation errors. The idea of jointly equalizing and decoding of coded data over ISI channels based on the Jacobi algorithm leads to the proposed turbo equalization scheme, which is shown to achieve comparable performance with a reduced complexity compared to the existing filter based equalization schemes, and superior performance compared to frequency domain OFDM and SC-FDE. Numerical comparison indicates that the MMSE filtering is not necessary unless in severe ISI situation. The good performance and simplicity of this scheme makes it a feasible alternative for practical implementations. How to improve the convergence rate and performance of the turbo equalization scheme using improved Jacobi algorithms, e.g., Jacobi over relaxation, serial Guass-Seideal, will be the future research topic for the authors.

\section{ACKNOWLEDGEMENT}

This work was sponsored by the UK Engineering and Physical Sciences Research Council under grant number EP/D07827X/1. The authors would like to acknowledge its financial support.

\section{REFERENCES}

[1] C. Douillard, M. Jezequel, C. Berrou. "Iterative correction of intersymbol interference: turbo-equalization". European Transactions on Telecommunications, pp. 507-511, Sept. 1995.

[2] X. Wang, H. Poor. "Iterative (turbo) soft interference cancellation and decoding for coded CDMA". IEEE Transactions on Communications, vol. 47, pp. 1046-1061, July 1999. 
[3] M. Tuchler, R. Koetter, A. Singer. "Turbo equalization: principles and new results”. IEEE Transactions on Communications, vol. 50, pp. 754-767, May 2002.

[4] M. Tuchler, A. Singer, R. Koetter. "Minumum mean square error equalization using a priori information". IEEE Transactions on Signal Processing, vol. 50, no. 3, March 2002.

[5] C. Laot, A. Glavieux, Joel Labat. "Turbo equalization: adaptive equalization and channel decoding jointly optimized". IEEE Journal on Selected Areas in Communications, vol. 19, no. 9, Sept. 2001.

[6] Z. Wu, J. Coiffi. "Low-complexity iterative decoding with decision-aided equalization for magnetic recording channels". IEEE Journal on Selected Areas in Communications, vol. 19, no. 4, April 2001.

[7] S. Weinstein, P. Ebert. "Data transmission by frequency-division multiplexing using the discrete Fourier transform". IEEE Trans. on Commun., vol. 19, no. 3, pp. 628-634, Oct. 1971.

[8] H. Sari, G. Karam, I. Jeanclaude. "Transmission techniques for digital terrestrial TV broadcasting”. IEEE Commun. Mag., pp. 100-109, Feb. 1995.

[9] D. Falconer, S. Ariavisitakul, A. Benyamin-Seeyar, B. Edison. "Frequency domain equalization for single-carrier broadband wireless systems". IEEE Communications Magazine, pp. 58-66, April 2002.

[10] J. Proakis. Digital Communications, 4th edition, McGraw-Hill, 2000.

[11] F. Hsu. "Square root Kalman filtering for high-speed data received over fading dispersive HF channels". IEEE Transactions on Information Theory, vol. 28, pp. 753-763, Sept. 1982.

[12] IEEE 802.16 Working Group on Broadband Wireless Access Standards. available at http://grouper.ieee.org/groups/802/16/. 2002.

[13] V. Erceg. "An empirically based path loss model for wireless channels in suburban environments". IEEE JSAC, vol. 17, no. 7, pp. 1205-1211, July 1999.

[14] S. Kay. Fundamentals of statistical signal processing, Prentice Hall, NJ, 1998.

[15] O. Axelsson. Iterative Solution Methods, Cambridge University Press, 1994.

[16] P. Xiao, R. Carrasco, I. Wassell. "Performance analysis of conventional detection in BFWA systems". Proc. Second IFIP International Conference on Wireless and Optical Communications Networks, WOCN'2005, pp. 447-452, March 2005.

[17] P. Xiao, R. Carrasco, I. Wassell. "Estimation of FWA MIMO channels". Proceedings IEEE Information Theory Workshop, ITW'2006, pp. 641-645, Oct. 2008.

[18] V. Erceg et al. "Channel models for fixed wireless applications". IEEE 802.16a cont. IEEE 802.16.3c-01/29r4, June 2003.

[19] P. Robertson, E. Villebrun, P. Hoeher. "A comparison of optimal and sub-optimal MAP decoding algorithms operating in the log domain”. Proc. IEEE International Conference on Communications, pp. 1009-1013, 1995.

[20] V. Poor, S. Verdu. "Probability of error in MMSE multiuser detection", IEEE Transactions on Communications, vol. 43, pp. 858-971, May 1997.

[21] A. Viterbi. "An intuitive justification and a simplified implementation of the MAP decoder for convolutional codes". IEEE Journals on Selected Areas in Communications, vol. 16, no. 2, pp. 260-264, Feb. 1998.

[22] C. Laot, R. Bidan, D. Leroux. "Low-complexity MMSE turbo equalization: a possible solution to EDGE". IEEE Transactions on Wireless Communications, vol. 4, no. 3, pp. 965-974, May 2005.

[23] P. Frenger, P. Orten, T. Ottosson. "Convolutional codes with optimum distance spectrum". IEEE Communication Letters, vol. 3, no. 11, pp. 317-319, Nov. 1999.

[24] P. Frenger, P. Orten, T. Ottosson. "Code-spread CDMA using maximum free distance low-rate convolutional codes". IEEE Trans. on Communications, vol. 48, no. 1, pp. 135-144, January 2000.

[25] C. Laot, R. Bidan, D. Leroux. "Low-complexity MMSE turbo equalization: a possible solution to EDGE". IEEE Trans. on Communications, vol. 4, no. 3, pp. 965-974, May 2005.

[26] M. WItzke, S. Baro, F. Schreckenback, J. Hagenauer. "Iterative detection of MIMO signals with linear detectors". In Proc. Asilomar Conf. Signals, Systems, Computers, pp. 289-293, Nov. 2002. 
[27] I. Chatzigeorgiou, M. Rodrigues, I. Wassell, R. Carrasco. "Turbo coded OFDM/SC-FDE techniques for MIMO BFWA channels". In Proc. International Symposium on Broadband Communications (ISBC), Dec. 2004.

[28] S. Brink. "Convergence behavior of iterative decoded parallel concatenated codes". IEEE Transactions on Communications, vol. 49, no. 10, pp. 1727-1737, Oct. 2001.

[29] S. Lee, A. Singer, N. Shanbhag. "Linear turbo equalization analysis via BER transfer and EXIT charts". IEEE Transactions on Signal Processing, vol. 53, no. 8, pp. 2883-2897, August 2005.

[30] P. Xiao, R. Carrasco, I. Wassell. "EXIT chart analysis of space-time turbo equalizer". Proc. IEEE Information Theory Workshop, ITW'2006, pp. 631-635, Oct. 2006.

[31] W. Webb. "Broadband fixed wireless access as a key component of the future integrated communications environment". IEEE Communication Magazine, vol. 39, pp. 115-121, Sept. 2001. 